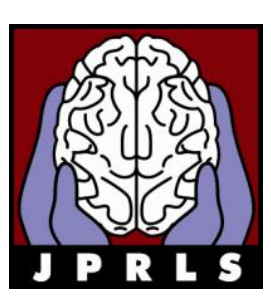

Open Access via www.jprls.org

Journal of Parkinsonism \&

\title{
Subacute presentation of a large focal lesion: A case and literature review
}

\author{
Bharat Bhushan, Lawrence A. Zumo* \\ Prince George's Hospital Center, Department of Medicine, Cheverly, Maryland, USA
}

\begin{abstract}
Multiple sclerosis has several clinical subtypes, variants and mimickers. This can be diagnostically very perplexing by the very broad nature of its differential diagnosis. However with a high index of suspicion, the differential can be rapidly narrowed and the correct clinical diagnosis deduced and targeted therapy instituted rapidly in order to spare the patient unnecessary resections and potentially debilitating procedures or adjunctive therapies as is demonstrated in this present case of tumefactive multiple sclerosis in a young healthy male presenting with subacute right hemiparesis and dyesthesia, whose clinical investigations are herein reported.
\end{abstract}

\section{INTRODUCTION}

Multiple sclerosis (MS), the most common of the chronic inflammatory demyelinating diseases, is characterized by destruction of the normal myelin with MS plaques having predilection for periventricular white matter, optic tracts, internal capsule, corpus callosum, pons and brachium pontis and spinal cord. The standard diagnosis of multiple sclerosis is based on clinical, laboratory, and/or radiographic evidence of disease separated in space

\footnotetext{
* Correspondence to: Lawrence Zumo, MD, 1111 Spring St, Suite 216, Silver Spring, MD 20910, Email: zumoamos@aol.com, Telephone: 1-301-562-8400, Fax: 1-301-562-8930.
}

or time. ${ }^{12}$ Other demyelinating lesions include acute disseminated encephalomyelitis with characteristic symmetric subcortical white matter lesions bihemispherically; central pontine myelinosis with its usual sparing of the periphery of the pons; radiation endarteritis, radiation necrosis, and the leukodystrophies (including Krabbe's, metachromatic leukodystrophy, adrenoleukodystrophy) which can be fairly easily distinguished because of their clinical features, course and radiographic characteristics. Clinical types of multiple sclerosis include relapsing remitting, primary progressive, secondary progressive and progressive remitting types. Relapsing remitting multiple sclerosis with main lesions in the optic nerve and spinal cord are classified as optic-spinal multiple sclerosis (OSMS). Balo's concentric sclerosis and Devic's neuromyelitis optica and Marburg variant are the other types of MS that are encountered in the clinical neurology literature. The spectrums of diseases that can mimic multiple sclerosis are numerous and are important to recognize as the treatment paradigm are different for each of these diseases. These include: primary CNS vasculitis, neoplasms (lymphoma, glioma, gliobastoma multiforme), cerebral abscesses, CNS toxoplasmosis, cystersiscosis, Lyme disease, antiphospholid antibody syndrome, human immunodeficiency virus (HIV), sarcoidosis, HTLVI/II infection (tropical spastic paraparesis), mitochondrial encephalopathy with lactic acidosis and stroke (MELAS), ischemic optic neuropathy (arteritic and nonarteritic), cerebral autosomal dominant arteriopathy, subcortical infarcts, and leukoencephalopathy (CADASIL), Behcet's disease, 
Macharfavi-Bigmani disease, progressive multifocal leukoencephalitis, stroke and ischemic cerebrovascular disease, Vitamin B12 deficiency, systemic lupus erythematosis.

We report the results of our investigations of a perplexing tumor-like presentation of a young male who presented at our institution with subacute onset of right hemiparesis and dyesthesia of unclear etiology. As this was a retrospective case evaluation and review of the literature, Institutional Review Board permission was not necessary.

\section{METHODS}

\section{Case report}

A 29 years old male (HIV negative, non-illicit drug abuser) with no significant past medical history except herniorrhaphy in childhood but with strong family history of multiple sclerosis (father, aunt, sister) presented to the emergency department of our institution (a Level 2 trauma midsize community hospital center) with complaints of pain and progressive numbness and weakness of right upper and lower limb of two weeks duration and acute gait imbalance right before admission. He also reported a $15 \mathrm{lbs}(6.8 \mathrm{~kg})$ weight loss over a two week period. He denied any fall, head or extremity trauma, fever, chills, rigors, diarrhea, headache, nausea, vomiting, visual loss nor obscuration, bladder or bowel incontinence. No recent travels abroad nor any high risk sexual behavior or illicit drug abuse. He was afebrile and the rest of his vitals were within normal limits. Physical examination revealed a male of normal habitus with unremarkable features. Neurological examination disclosed a normal mental status. Cranial nerve examination revealed intact cranial nerves except mild right afferent pupillary defect. He had preserved motor strength on the left hemibody but had moderate weakness on the right upper extremity (MRC Grade 3/5) and in the right lower extremity (MRC Grade 4/5). The deep tendon reflexes were normal $(2+)$ in all four extremities. Plantar responses were flexor bilaterally. Sensory examination revealed intact light touch, pain pinprick, proprioception, temperature and vibration in all four extremities. Coordination examination revealed slow rapid alternating movements on the right but intact finger to nose but impaired heel to shin testing as well as inability to stand unassisted. Gait was not wide based but with tendency to fall on the right. Romberg's test was negative.

\section{Laboratory investigations}

The following pertinent laboratory investigations were normal or negative: serologic assay for HIV, anti nuclear antibody, serum toxoplasmosis titer, serum lyme titer, serum cystercercosis titer, anti-extractable nuclear antigen, erythrocyte sedimentation rate, platelet count, urine toxicology, liver and renal panels, thyroid function studies and serum angiotensin converting enzyme levels.

The following initial laboratory investigations were either abnormal or significant: White blood cell count $4.4 \mathrm{~K}$ (nl range $4.5-10.5$ ), hemoglobin 12.6 $\mathrm{g} / \mathrm{dl}$ (nl range 13-17), hematocrit $37.4 \%$ (nl range 39-51), eosinophils $6.6 \%$ (nl 1-6).

Lumbar puncture was not done.

\section{Neuroimaging studies}

The CT scan of the head revealed prominent area $(3.7 \mathrm{~cm})$ of deceased attenuation within the left upper parietal lobe (figure 1). This area is within the centrum semiovale and extends to the cortex with mild gyral effacement suggesting vasogenic edema but with no midline shift. Carotid duplex ultrasonography did not reveal any hemodynamically significant stenosis nor carotid dissection and $2 \mathrm{D}$ Echocardiography did not reveal any valvular lesions nor intracardiac thrombus or vegetations. A 1.5 Telsa MRI scan of the brain was performed in sagittal, axial and coronal plans with and without intravenous gadolinium enhancement (figures 2, 3, 4, 5). Thin sections in the axial, sagittal and coronal plan revealed a rounded mass in the left frontal lobe posteriorly involving the left superior centrum semiovale. The mass measured about 2.5 to $3 \mathrm{~cm}$ in diameter with extensive surrounding edema. It revealed low signal intensity in the $\mathrm{T} 1$ weighted pulse sequence and increased signal intensity in T2 weighted pulse sequence. There was extensive surrounding edema which was mostly vasogenic. On diffusion weighted pulse sequence, the peripheral edema reveals increased signal intensity with central low intensity in the area of the mass. On the apparent diffusion coefficient (ADC) map, the signals were reversed with high signals in the mass and somewhat decreased signals intensity in the surrounding edema. The mass revealed arc-like ring enhancement on the post contrast images with the periphery of the mass enhancing with contrast material. The edema was unchanged and revealed no enhancement. The central portion of the mass was low in signal and revealed no contrast enhancement on the post contrast $\mathrm{T} 1$ weighted pulse sequence. No significant mass effect was seen nor any ventricular compression or midline 
shift. No additional lesions were identified in the brain parenchyma. Radiologically the differential diagnosis included toxoplasmosis, lymphoma, brain abscess, primary brain tumor or astrocytoma.

MRI lumbar spine revealed degenerative disc disease with posterior and left paracentral protrusion of the L5-S1 intervertebral disc abutting the left L5 nerve root but no cord compression nor cord edema or abscess. MRI of the thoracic and cervical spine were essentially normal.

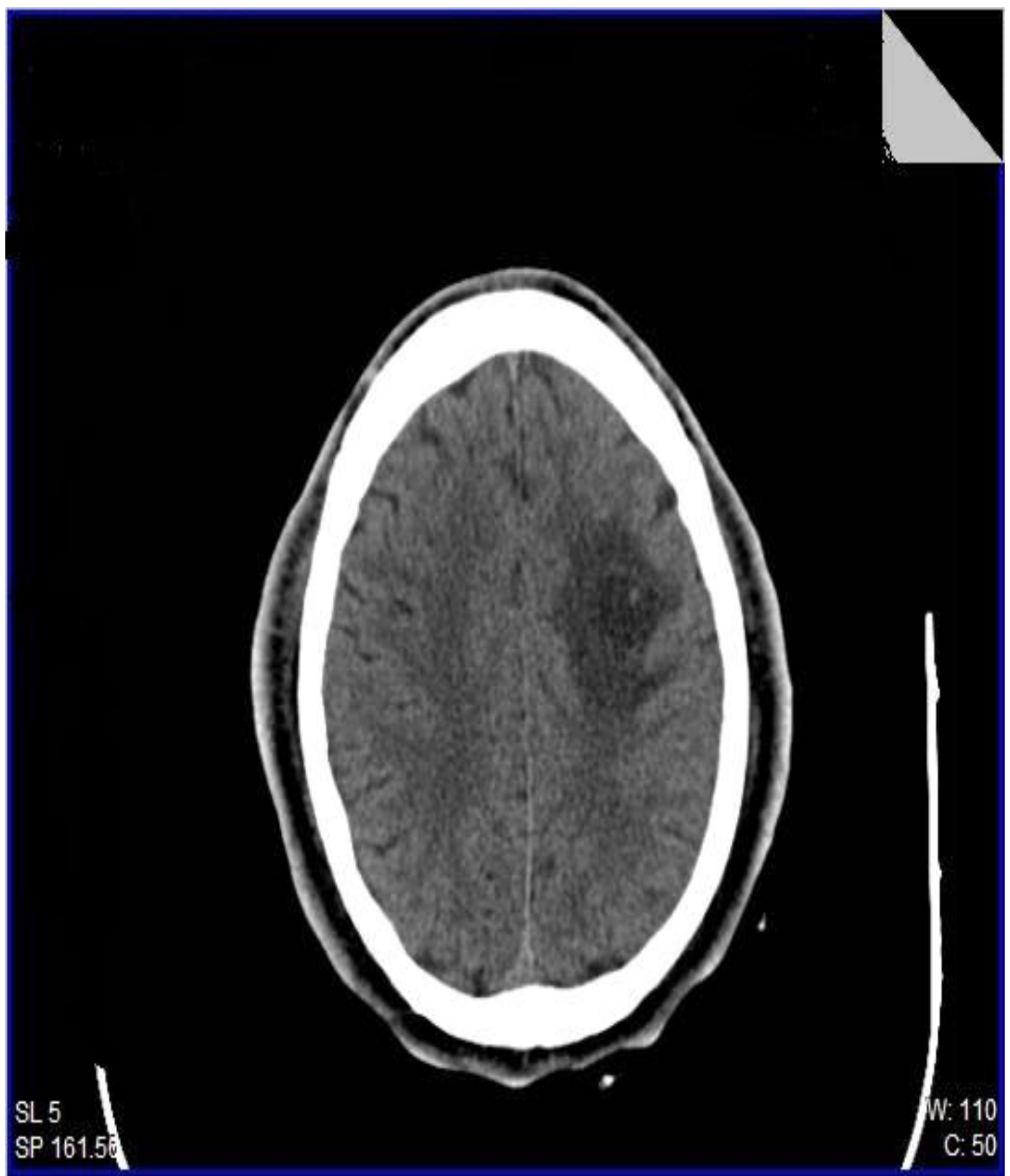

Figure 1. Non-contrast CT head showing prominent area of decreased attenuation with vasogenic edema in the centrum semiovale in the left upper parietal lobe. 


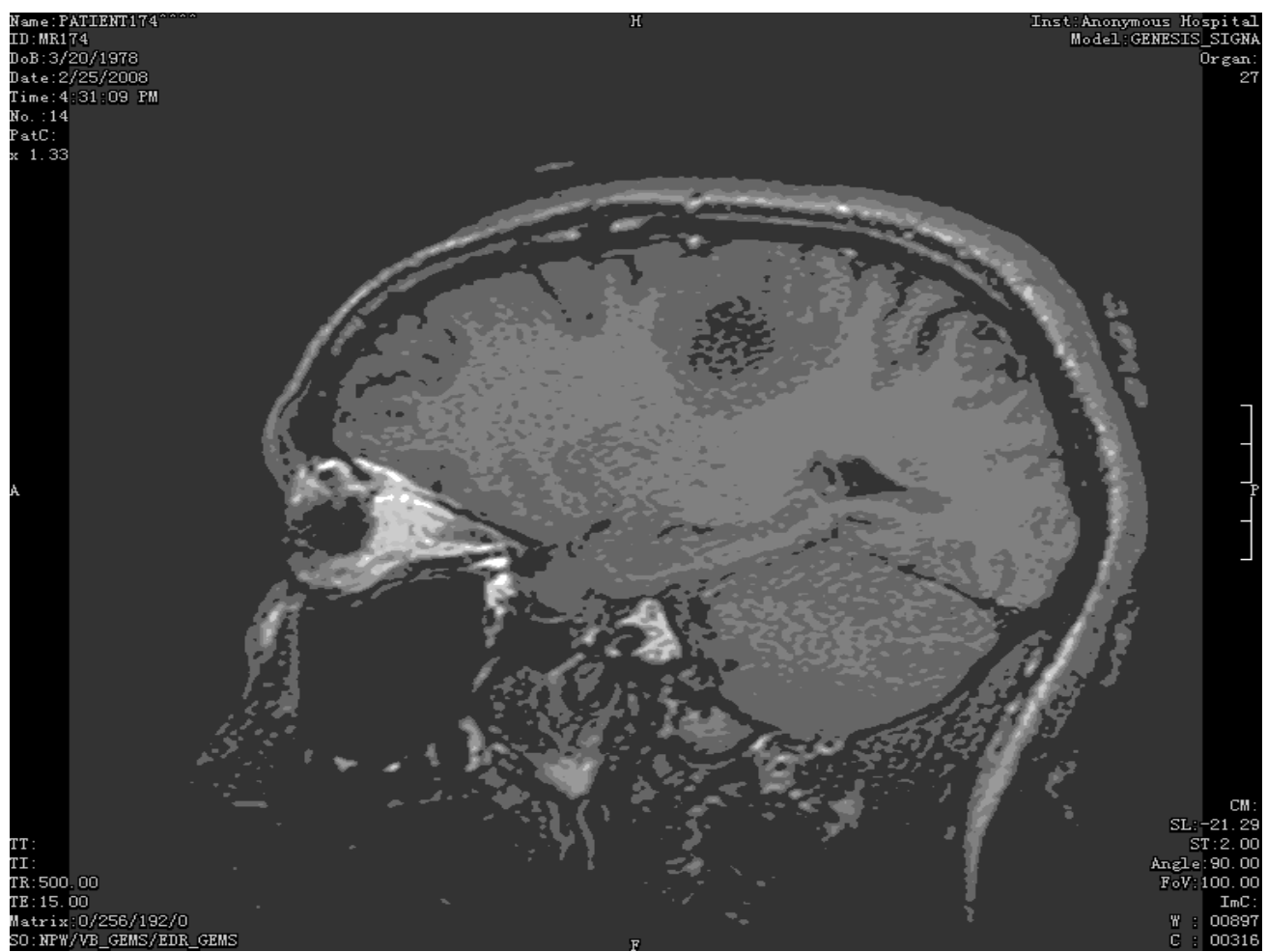

Figure 2. Sagittal T1 weighted MRI without contrast reveals a rounded mass in the left frontal lobe posteriorly involving the left superior centrum semiovale. The mass measure $2.5-3 \mathrm{~cm}$ in diameter with surrounding vasogenic edema.

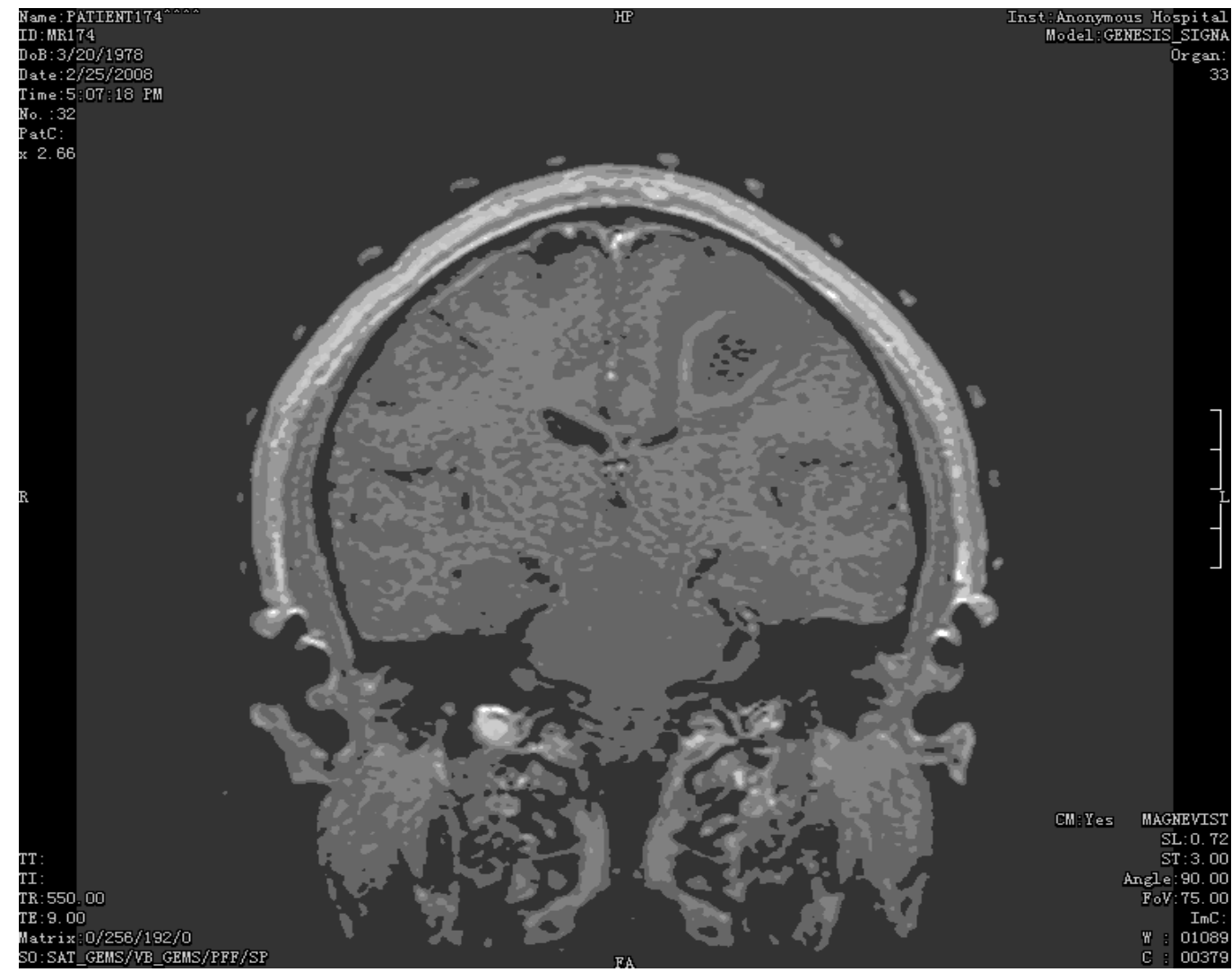

Figure 3. Coronal T1 weighted MRI film with contrast, revealing ring enhancement with the periphery of the mass enhancing as well. 


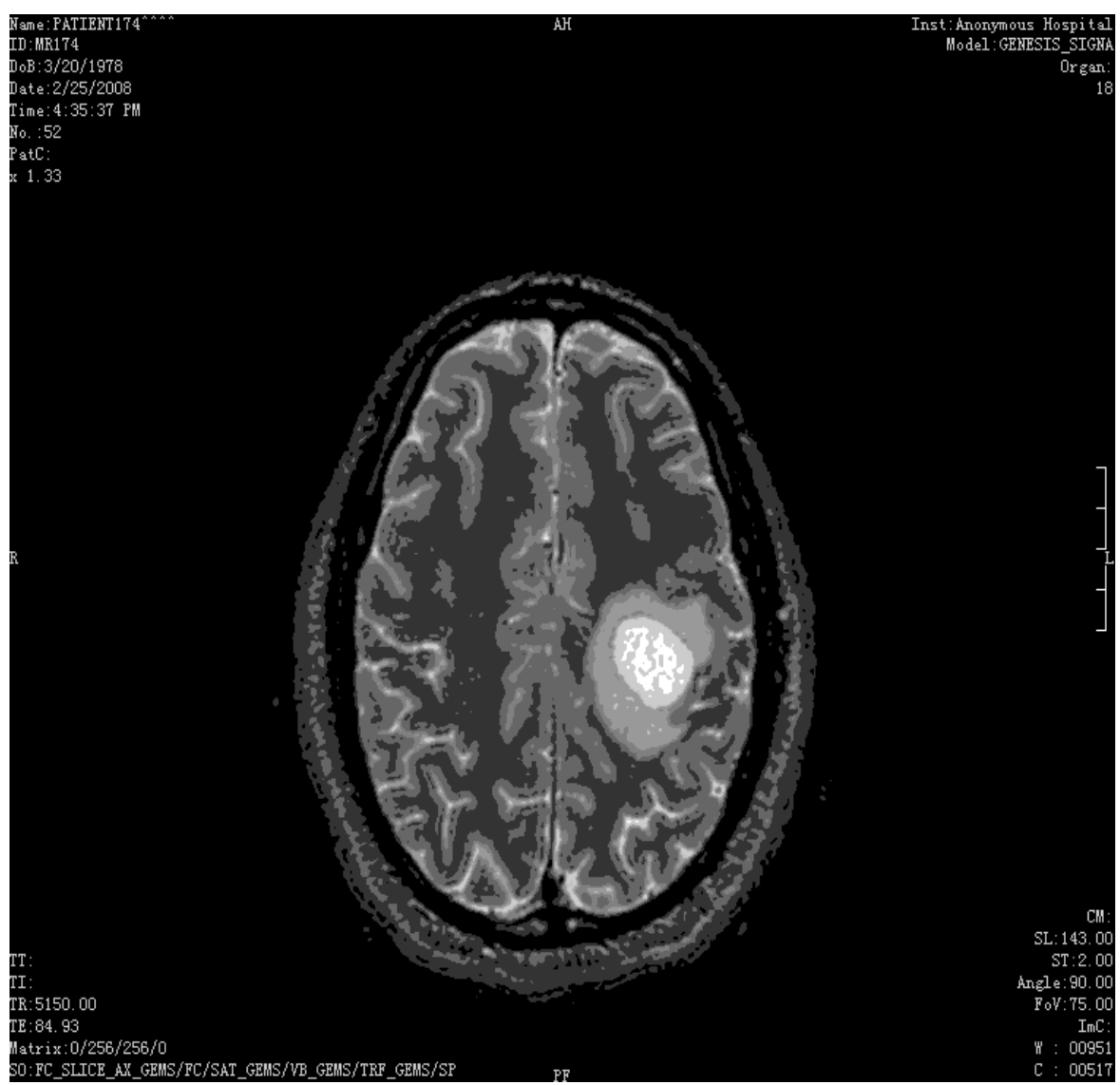

Figure 4. Axial T2 weighted MRI without contrast revealing mass with surrounding edema which is mostly vasogenic.

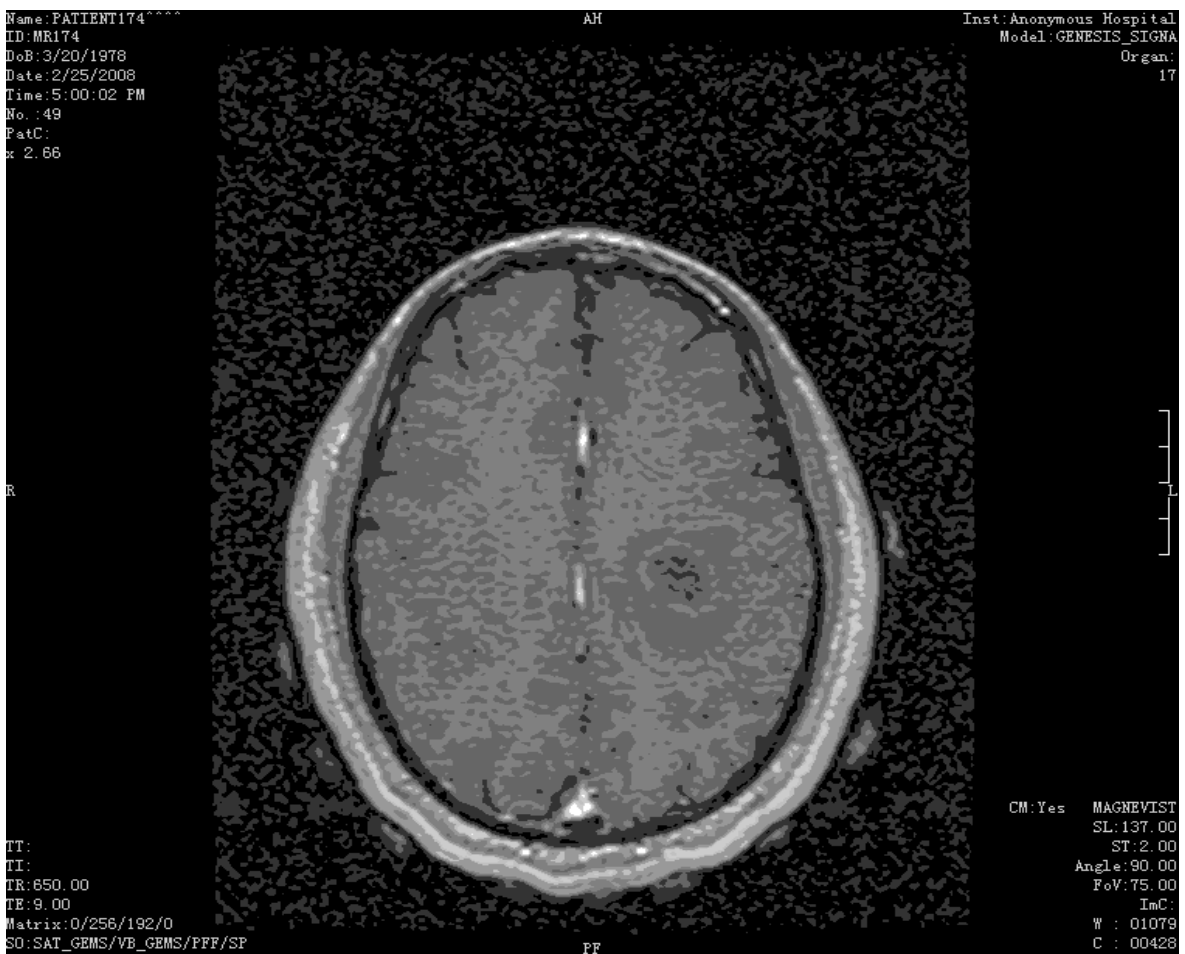

Figure 5. Coronal T1 weighted MRI film with contrast, revealing ring enhancement with the periphery of the mass enhancing as well. 


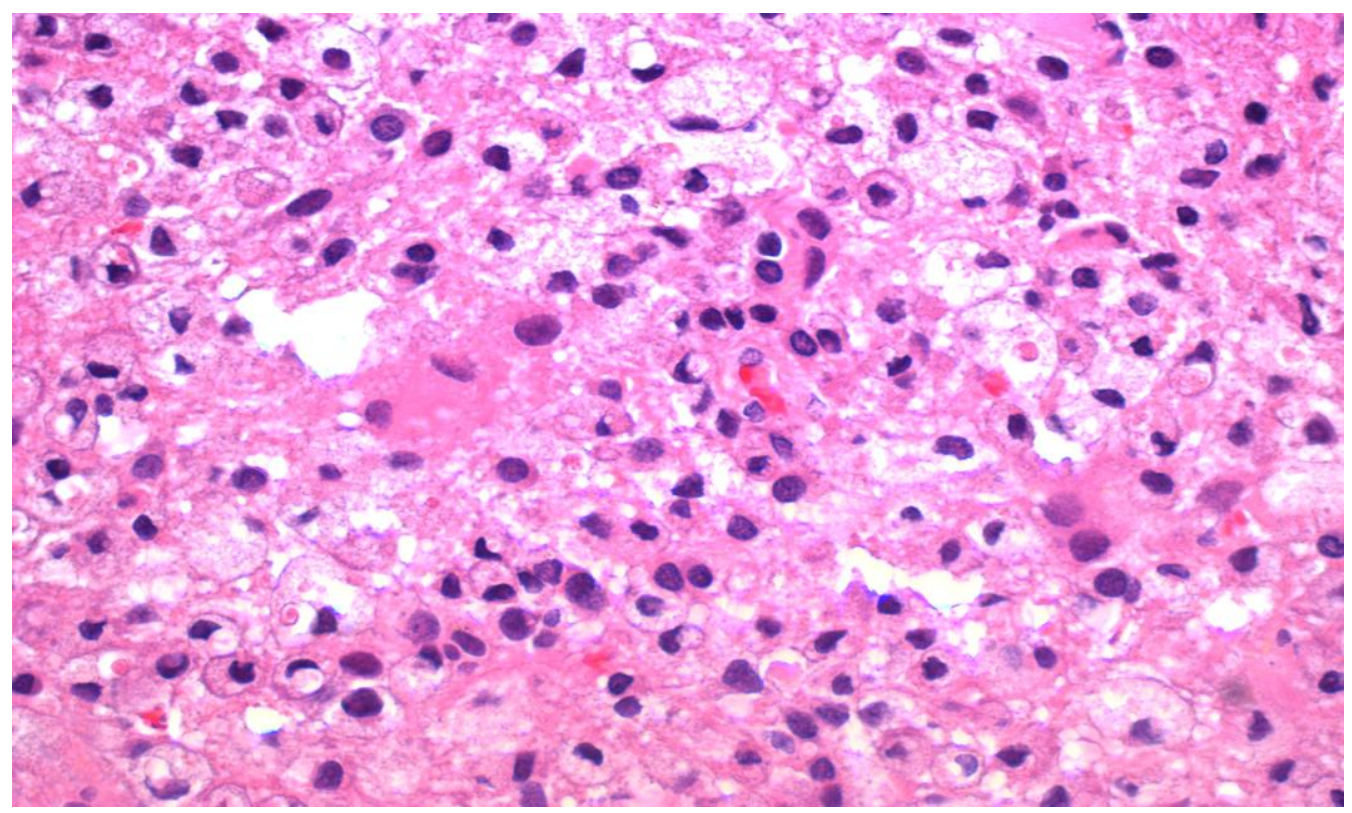

Figure 6. Axial T1 weighted MRI with contrast reveals mass with ring enhancement on the post contrast image. The edema in unchanged and reveals no enhancement.

\section{DisCUSSION}

Our case emphasizes the importance of having a high index of clinical suspicion and a broad differential diagnostic acumen when facing the myriad of illnesses that present daily in busy hospitals and emergency rooms in countries the world over so as to ensure the correct and rapid clinical diagnosis for the earliest appropriate therapeutic intervention whilst avoiding unnecessary debilitating adjunctive therapy with untold side effects and residuals. Multiple sclerosis is usually diagnosed by demonstrating clinical and or radiographic evidence of dissemination of disease in time and space. ${ }^{12}$

Primary demyelinating disease of the central nervous system (CNS) generally does not produce a focal diffuse mass lesion, a feature that has been used to distinguish demyelinating lesions from tumors. ${ }^{4}$ Although the diagnosis of classic multiple sclerosis generally does not require surgical intervention, some cases do pose considerable diagnostic difficulties and may require brain biopsy, as was done in our case. ${ }^{3}$ (figures 6,7)

Atypical imaging features of multiple sclerosis lesions include size more than $2 \mathrm{~cm}$, mass effect, peri-lesional edema and or ring enhancement. This constellation is often referred to as 'tumefactive multiple sclerosis. ${ }^{1011} 1213$ The occurrence of tumorlike demyelination is reportedly rare, being estimated at $1-2 / 1000$ cases of multiple sclerosis. $^{14} \mathrm{~A}$ prevalence of three cases per million inhabitants per year has been suggested. ${ }^{15}$ Clinical presentations are dependent on lesion location and size, and as a result are variable and include headache, cognitive abnormalities, mental confusion, aphasia, apraxia and/or seizures. Magnetic resonance imaging findings of CNS glioma may also look similar to those of multiple sclerosis with T2-hyperintense lesions in the cerebral white matter extending into corpus callosum and variable gadoliniumenhancement. ${ }^{16}$

Tumefactive demyelinating lesions tend to be circumscribed lesions with little mass effect or vasogenic edema. ${ }^{4 \text { (figures } 3,4,5)}$ Approximately half of tumefactive demyelinating lesions have pathologic contrast enhancement, usually in the form of ring enhancement. ${ }^{4}{ }^{17}$ Commonly the enhancement patterns will be in the form of an open ring, with the incomplete portion of the ring on the gray matter side of the lesion. ${ }^{17}$ More typical active multiple sclerosis plaques exhibit this open-ring or arc like pattern of enhancement only $9 \%$ of the time. The enhancing portion of the ring is believed to represent the leading edge of demyelination and thus favors the white matter side of the lesion. ${ }^{18}$ The central non-enhancing core represents a more chronic phase of the inflammatory process. Tumefactive demyelinating lesions have been misinterpreted as gliomas, with the correct diagnosis being revealed only after histologic evaluation. ${ }^{426}$ The most common neoplasm mistaken for MS is CNS lymphoma. ${ }^{27}$

Histologic features may mimic tumor including hypercellularity, astrocytic pleomorphism, variable nuclear atypia, a rare mitotic figure and occasional necrosis or cystic changes. ${ }^{28} 2930$ These features, 
particularly at frozen section or on small biopsies, pose a diagnostic hurdle for the pathologist. Several histologic features, however, do point toward a nonneoplastic process. These include (i) abundance of foamy macrophages in the absence of coagulative necrosis, (ii) evenly distributed plump, reactive astrocytes, some with multiple micronuclei (Creutzfeldt cells), often closely intermingled with macrophages,(iii) absence of microvascular proliferation, (iv) perivascular inflammation and (v) relative axonal preservation. ${ }^{28} 2930$

In a review of 31 cases, Kepes ${ }^{35}$ proposed that tumefactive demyelinating lesions represent an intermediate lesion between those typically seen with multiple sclerosis and acute disseminated encephalomyelitis, typically a monophasic illness with a favorable long term prognosis. Pathologically, these lesions are indistinguishable from typical multiple sclerosis plaques and are characterized by infiltrating foamy macrophages intermingled between reactive astrocytes. Significant quantities of lipid may accumulate within the plaques as a result of myelin breakdown and its intermediate metabolites. The axons are relatively preserved within the lesions, but more recent investigations seem to suggest axonal injury as well with multiple sclerosis plaques. The pathologic diagnosis may be challenging based on the initial frozen-section specimen when the primary suspicion is malignancy. However, ancillary histopathologic and immunochemical assays can point to the correct diagnosis. ${ }^{36}$

A variety of intracranial pathologies can present as a ring enhancing lesion on MRI, including glioma, metastasis, lymphoma, radiation necrosis, infarct, abscess and IDD. In a recent series, the most prevalent pathologies associated with ring enhancement were gliomas $(40 \%)$, metastasis $(30 \%)$, abscesses $(8 \%)$ and multiple sclerosis lesions $(6 \%) .^{13}$

The radiographic distinction may be made by the demonstration of involvement of gray matter structures out of proportion to white matter; however lesion behavior over time is more helpful. Enhancement of MS lesions rarely persists for longer than 4-6 weeks. Thus, persistent Gadoliniumenhancement, particularly if there is a prominent component of enhancement along blood vessels, and continued enlargement of lesions strongly suggests a neoplasm. Ultimately, biopsy is necessary to make the correct diagnosis. ${ }^{19}$

The MRI appearance of these tumefactive demyelinating lesions can aid in preoperative diagnosis and assist with the final pathological interpretation (figures 4, 5). The most useful neuroimaging diagnostic tool is reported to be the open ring enchancement (primarily differentiating it from an abscess and intraparenchymal infectious cysts) and relatively sparse mass effect and vasogenic edema associated with these often sizeable lesions, as was in our case. The presence of centrally dilated veins within the lesion and decreased perfusion appear to be additional characteristic features. ${ }^{34}$

Modern MR imaging is the most sensitive method of detecting the white matter lesions of multiple sclerosis. T2W MRI displays multiple, well demarcated, ovoid lesions, which, within periventricular white matter and the corpus callosum, are often oriented perpendicular to the long axis of the ventricular system ${ }^{57}$ Although MRI has increased the ability to highlight these lesions, it often fails to provide an unambiguous diagnosis. ${ }^{8}$ This is particularly true when the lesions present as large, space occupying lesions misinterpreted as tumor, abscess or infarct. Furthermore, some malignancies, such as metastases, primary CNS lymphoma, and intraparenchymal cysts may be characterized by multiple small lesions with variable or persistent enhancement patterns (nodular, punctate, etc).

MR abnormalities are best demonstrated using T2 weighted or fluid attenuated inversion recovery (FLAIR) images, which typically demonstrate hyperintense white matter lesions with a predilection for periventricular areas and the corpus callosum. Occasionally, however, atypical plaques can develop, which because of their size, position, mass effect and surrounding vasogenic edema, mimic intra-cerebral tumors clinically, radiologically and even histopathologically. $^{49}$

Single voxel MR Spectroscopy is commonly used to identify five major chemical resonances in vivo. ${ }^{9}$ These are choline containing small molecules, including phospholipids (CHO), involved in the synthesis of cellular membranes (MR resonance 3.2 parts per million ); phosphocreatine and creatine (CR), involved in cellular metabolism ( MR resonance $3.0 \mathrm{ppm}$ ); $\mathrm{N}$-acetyl aspartate (NAA), a neuronal marker thought to act as a neurotransmitter (MR resonance $2.0 \mathrm{ppm}$ ); lactate (LAC), a product of anaerobic glycolysis and indirect marker of ischaemic tissue (MR resonace $1.3 \mathrm{ppm}$ ); and lipids (LIP), which are a marker of cell membrane breakdown (MR resonace $0.9 \mathrm{ppm}$ ).

Proton MR spectroscopy provides insight into the chemical composition of lesions. Primary glial cell tumors produce a characteristic spectrum consisting of elevated choline with suppressed levels of $\mathrm{N}$-acetylaspartate. Additionally, there may be detectable levels of lipids and lactate corresponding to necrosis and anaerobic metabolism associated with the glial tumors (primarily glioblastoma multiforme). Several non-neoplastic brain lesions (including tumefactive demyelinating lesions) may produce an 
identical MR spectrum, mimicking a neoplastic process, as was in our case. ${ }^{2021}$

Low grade gliomas have been consistently shown to exhibit reductions in NAA and increases in $\mathrm{CHO}$ resonance intensity. These findings reflect the replacement of normal neuronal tissue with a proliferating cellular process. ${ }^{22}$

Increases in LAC (lactate) are not uncommon, as a result of tissue ischemia secondary to abnormal angiogenesis and edema within and surrounding the tumor. $^{2324}$

Variable increases in LIP have also been described. $^{25}$

Although, Proton MR spectroscopy is a powerful noninvasive tool for understanding the biochemical alterations in various intracranial disease processes, Saidane et al found in their study that high-grade gliomas and TDLs (Tumeafective demylenating diseases) share many similar features at both conventional MR imaging and proton MR spectroscopy. Except for significant differences in the $\mathrm{NAA} / \mathrm{Cr}$ ratio in their central regions, no other metabolite ratios could be used to distinguish highgrade gliomas and TDLs with confidence. These findings emphasize the need for the cautious interpretation of spectroscopic findings and cannot solely be used to distinguish high grade gliomas from TDL's. ${ }^{22}$

\section{CONCLUSION}

Tumefactive demyelinating lesions, thus, can pose a very significant diagnostic dilemma to clinicians, neurosurgeons, radiologists, and pathologists. The preoperative diagnosis, or at least consideration, of a demyelinating process is imperative in the intial differential diagnosis consideration. ${ }^{4}$

The histopathologic characteristics of demyelinating lesions which include the preservation of axons as shown by the presence of neurofilaments, the loss of myelin as revealed by negative luxol fast blue staining, the presence of macrophages as depicted by the presence of CD-68, and astrocytic proliferation as manifested by the presence of glial fibrillary acidic protein leads to correct diagnosis. Suboptimal tissue sampling also represents a major impediment to accurate histological diagnosis. A good sample should include perilesional brain tissue and not be entirely subject to frozen section; smear preparations are preferred. Standard tumour histopathological protocols do not include staining with Luxol fast blue, which is used to detect myelination. Therefore, it is important for the clinician to have a high index of suspicion for a demyelinating lesion and relay this to the neuropathologist. ${ }^{31}$ Distinguishing active demyelination from neoplasm is critical, since a misdiagnosis can lead to inadvertent brain irradiation, which apart from the potential risk of radiation necrosis and postirradiation neoplasia, is known to exacerbate underlying inflammatory demyelinating disease. ${ }^{32} 33$

The case herein presented demonstrates the importance of considering a demyelinating disease in the differential diagnosis of a tumor-like brain lesion in the clinical setting as the management and therapeutic intervention as well as clinical outcomes are quite different. Arriving at the definitive diagnosis early and rapidly can potentially spare the patient unnecessary and possibly debililating surgical resection and other procedures and therapies. Larger, possibly multicenter clinical, magnetic resonance, spectroscopic studies and advances are indicated to further our understanding of the pathophysiology, clinical course and natural progression of tumefactive demyelinating disease lesions.

\section{ACKNOWLEDGEMENTS}

Many thanks to Joel Falik, MD for the neurosurgical stereotatic biopsy, Dr Manlachu et al for histopathology and immunohistochemistry, G. Siddhu, MD for radiology services , and Manash Das, MD, Teshome Tegene, MD, Linda Green, MD, ( all of the Department of Internal Medicine, Prince George's Hospital Center, Cheverly, MD).

\section{REFERENCES}

1. Poser CM, Paty DW, Scheinberg L, McDonald WI, Davis FA, Ebers GC, et al. New diagnostic criteria for multiple sclerosis: guidelines for research protocol. Ann Neurol 1983; 13:227-231

2. McDonald WI, Compston A, Edan G,Goodkin D, Hartung HP, Lublin FD, et al. Recommended diagnostic criteria for multiple sclerosis. Ann Neurol 2001; 50:121-127

3.Lucchinetti CF, Gavrilova RH, Metz I, Parisi JE ,Scheithauer BW, Weigand S, et al Clinical and radiographic spectrum of pathologically confirmed tumefactive multiple sclerosis. Brain2008; 131:17591775

4. Dagher AP, Smirniotopoulos J. Tumefactive demyelinating lesions. Diagnostic Neuroradiol 1996; 38: 560-565

5.Barkhof F,Fillippi M,Miller DH,Scheltens P,Campi A,Polman $\mathrm{CH}$, et al.Comparison of MRI criteria at first presentation to predict conversion to clinically definite multiple sclerosis. Brain 1997; 120:20592069 
6. Fazekas F,Barkhof F,Fillippi M,Grossman RI,Li DK,McDonald WI, et al.The contribution of magnetic resonance imaging to the diagnosis of multiple sclerosis. Neurology 1999; 53:448-456

7.Miller DH, Fillippi M, Fazekas F, Frederiksen JL,Matthews PM,Montalban X, et al. Role of magnetic resonance imaging within diagnostic criteria for multiple sclerosis. Ann Neurol 2004; 56:273-278

8.Butteriss DJ,Ismail A,Elison DW,Birchall.Use of serial proton magnetic resonance spectroscopy to differentiate low grade glioma from tumefactive plaque in the patient with multiple sclerosis. $\mathrm{Br} \mathrm{J}$ Radiol 2003;76:662-665

9. Hunter SB, Ballinger WE Jr, Rubin JJ. Multiple sclerosis mimicking primary brain tumour. Arch Pathol Lab Med 1987; 111:464-468

10.Sagar HJ, Warlow CP, Sheldon PWE, Esitiri MM. Multiple sclerosis with clinical and radiological features of cerebral tumor.J Neurol Neurosurg Psychiatry 1982;45:802-808

11.Giang DW, Poduri KR, Eskin TA, Ketonen LM, Friedman PA,Wang DD, et al. Multiple sclerosis masquerading as a mass lesion. Neuroradiology 1992; 34:150-154

12. Al-Bunyan MA. Tumor-like presentation of multiple sclerosis. Saudi Med J 2000; 21:393-395

13.Schwartz KM, Ericson BJ, Lucchinetti CF. Pattern of T2 hypointesnity associated with ring enhancing brain lesion can help differentiate pathology. Neuroradiology 2006; 48:143-149

14. Poser S, Luer W, Bruhn H, Frahm J, Bruck Y, Felgenhauer K. Acute demyelinating disease.Classification and non-invasive diagnosis. Acta Neurol Scand 1992; 86:579-585

15. Paty DW, Oger JJ, Kastrukoff LF, Hashimoto SA, Hooge JP, Eisen AA,et al. MRI in the diagnosis of MS: a prospective study with comparison of clinical evalution, evoked potentials, oligoclonal banding and CT. Neurology 1988;38:180-185

16.Kalyan-Raman UP, Garwacki DJ, Elwood PW. Demyelinating disease of corpus callosum presenting as glioma on magnetic resonance scan: A case documented with pathological findings. Neurosurgery 1987; 21: 247-250

17. Masdeu JC, Moreira J, Trasi S, Visintainer P, Cavaliere $\mathrm{R}$, Grundman M. The open ring: a new imaging sign in demyelinating disease. J Neuroimaging 1996; 6:104-107

18.He J, Grossman RI, Ge Y, Mannon LJ. Enhancing patterns in multiple sclerosis: evolution and persistence. Am J Neuroradiol 2001; 22:664-669

19.Nelson MJ, Miller SL, McLain LW Jr, Gold LG. Multiple sclerosis: Large plaque causing mass effect and sign. J Comput Assist Tomogr 1981; 5: 892-894

20.Ernst T, Chang L, Walot I, Huff K. Physiologic MRI of a tumefactive multiple sclerosis lesion. Neurology 1998; 51:1486-1488

21. Saindane AM, Soonmee C, Meng L, Xiaonan X, Knopp EA, Zagzag D. Proton MR spectroscopy of tumefactive demyelinating lesions. Am J Neuroradiol 2002; 23:1378-1386

22. Moller H, Kneller RW, Boice JD, Olsen JH. Cancer incidence following hospitalization for multiple sclerosis in Denmark. Acta Neurol Scand 1991; $84: 214-220$

23.Poptani H, Gupta RK, Roy R, Pandey R, Jain VK, Chhabra DK. Characterization of intracranial mass lesions with in vivo proton MR spectroscopy. Am J Neuroradiol 1995; 16: 1593-1603

24. Preul MC, Caramanos Z, Collins DL, Villemure JG, Leblanc R, Olivier A, et al. Accurate, noninvasive diagnosis of human brain tumors by using proton magnetic resonance spectroscopy. Nat Med 1996; 2:323-235

25. Rand SD, Prost R, Haughton V, Mark L, Strainer J, Johansen $\mathrm{J}$, et al. Accuracy of single-voxel proton MR spectroscopy in distinguishing neoplastic from nonneoplastic brain lesions. Am J Neuroradiol 1997; 18:1695-1704

26. Paley RJ, Persing JA, Doctor A, et al. Multiple sclerosis and brain tumor: a diagnostic challenge. J Emerg Med 1989; 7:241-244

27. DcAngelis LM. Primary central nervous system lymphoma imitates multiple sclerosis. J Neurooncol 1990; 9: 177-181

28.Zagzag D, Miller DC, Kleinman GM, Abati A, Donnenfeld H, Budzilovich GN. Demyelinating disease versus tumor in surgical neuropathology. clues to a correct pathological diagnosis. Am J Surg Pathol 1993; 17:537-545

29.Annesley-Williams D, Farrell MA, Staunton H, Brett FM. Acute demyelination, neuropathological diagnosis and clinical evolution. J Neuropathol Exp Neurol 2000;59:477-489

30.Sugita Y,Terasaki M,Shigenori M,Sakata K,Morimatsu M.Acute focal demyelinating disease simulating brain tumors: Histopathological guidelines for an accurate diagnosis.Neuropathology 2001;21:25-31

31. Aria Fallah, Sarfaraz Banglawala, Shanil Ebrahim, John E. Paulseth, Neilank K. Jha. Can J Surg 2010; 53:60-70

32.Peterson K, Rosenblum M, Powers J, Alvord E, Walker R, Posner J. Effect of brain irradiation on demyelinating lesions. Neurology 1993; 43:21052112

33.Miller RC, Lachance DH, Lucchinetti CF, Brown PD, Keegan BM, Bhatia S, et al. Multiple sclerosis, brain radiotherapy, and risk of neurotoxicity: the Mayo clinic experience. Int.J Radiat Oncol Biol Phys 2006; 66:1178-1186

34. Given CA, Stevens BS, Lee C. The MRI appearance of Tumefactive Demyelinating lesions. American Journal of Roentgenology 2004: 182: 195-199

35. Kepes JJ. Large focal tumor-like demyelinating lesions of the brain; intermediate entity between multiple sclerosis and acute disseminated encephalomyelitis: A study of 31 patients. Ann Neurol 1993: 33: 18-27

36. Paley RJ, Persing JA, Doctor A, et al. Multiple sclerosis and brain tumor: a diagnostic challenge. J Emerg Med 1989; 7:241-2. 